\title{
Immunotherapy in non-small cell lung cancer: steps towards more effective combination therapies
}

\author{
Matthew W. Rosenbaum ${ }^{1}$, Mari Mino-Kenudson ${ }^{1,2}$ \\ ${ }^{1}$ Department of Pathology, Massachusetts General Hospital, Boston, MA, USA; ${ }^{2}$ Harvard Medical School, Boston, MA, USA \\ Correspondence to: Mari Mino-Kenudson, MD. Department of Pathology, Massachusetts General Hospital, 55 Fruit Street, Warren 122, Boston, MA \\ 02114, USA. Email: mminokenudson@partners.org. \\ Comment on: Yonesaka K, Haratani K, Takamura S, et al. B7-H3 Negatively Modulates CTL-Mediated Cancer Immunity. Clin Cancer Res \\ 2018;24:2653-64.
}

Submitted Jul 28, 2018. Accepted for publication Aug 02, 2018.

doi: $10.21037 /$ tcr.2018.08.08

View this article at: http://dx.doi.org/10.21037/tcr.2018.08.08

\section{Evolution of cancer treatment and immunotherapy for lung cancer}

Non-small cell lung carcinoma (NSCLC), one of the most common causes of cancer death worldwide (1), is also where some of the most progressive and innovating breakthroughs are being made in oncologic research. With the progression from single-agent chemotherapy to combination chemotherapy, targeted therapy, and into the most recent developments of immunotherapy and combination immunotherapy, these improvements in recent years have been groundbreaking. Each of these advancements has occurred paired with an improved understanding of tumor biology and clinical responses in lung cancer. Through this most recent generation of research, the possibility of unlocking the real potential of immunotherapy, mainly by targeting immune inhibitory molecules, has been realized.

The cancer immunity cycle consists of multiple steps, from the release of cancer cell antigens to cancer antigen presentation, priming and activation of $\mathrm{T}$ cells in lymph nodes, trafficking of $\mathrm{T}$ cells to tumors via blood vessels, infiltration of $\mathrm{T}$ cells into tumors, recognition of cancer cells by $\mathrm{T}$ cells, finally the killing of cancer cells in the tumor microenvironment (2). There are multiple inhibitory molecules/check points involved in each step. For instance, cytotoxic T-lymphocyte-associated antigen 4 (CTLA4) inhibits one of the important steps, priming and activation of $\mathrm{T}$ cells, by preventing the $\mathrm{B} 7$ protein on antigen presenting cells (APCs) from interacting with co-stimulatory CD28 found on T-cells (2). Similarly, vascular endothelial growth factor (VEGF), a molecule associated with angiogenesis and tumor growth, can create an immunosuppressive environment by preventing trafficking of $\mathrm{T}$ cells to tumors by inhibiting adhesion molecule expression on endothelial cells (3), and by recruiting regulatory $\mathrm{T}$ cells (4). Programmed cell death 1 (PD-1)/programmed deathligand 1 (PD-L1) inhibits cancer immunity mainly at the step of cancer cell killing (2), and currently, the majority of immunotherapy in NSCLC is related to disrupting the PD-1/PD-L1 axis (hereafter referred to as PD-1 blockade). $\mathrm{PD}-1$ is a receptor expressed on $\mathrm{T}$ cells that, on interacting with its ligand, PD-L1 (which may be expressed by tumor cells), causes T-cell anergy and apoptosis, allowing for immune evasion. Treatment with an anti PD-1 agent, pembrolizumab, has been shown to be superior to firstline chemotherapy in tumors that express PD-L1 on $\geq 50 \%$ of the tumor cells by immunohistochemistry (5). PD-1 blockade with pembrolizumab, another anti PD-1 agent, nivolumab, or an anti PD-L1 agent, atezolizumab, has also been shown to be a potentially effective later-line treatment for tumors with a lower level of PD-L1 expression (6-9). However, despite the general recognition of PD-1 blockade as a dramatic evolution in cancer therapy, only $44.8 \%$ of patients with high tumor PD-L1 expression had an initial objective response to first-line PD-1 blockade (5).

There are many potential reasons why a substantial proportion of patients with high PD-L1 expression do not respond to PD-L1 blockade, including host immune system, immune checkpoint co-expression and tumor microenvironment. In order to boost host immune response 
some recent trials have combined immunotherapy with conventional chemotherapy. For instance, in KEYNOTE 189, patients with NSCLC (EGFR/ALK wild type) were treated with platinum-based chemotherapy and were randomized to receive pembrolizumab or a placebo regardless of PD-L1 status. In their first interim analysis, it appears that the combination of immunotherapy and chemotherapy provided an across-the-board improvement of overall survival (OS) and progression-free survival (PFS) compared to chemotherapy alone $(\mathrm{HR}=0.49$ and 0.52 ; $\mathrm{P}<0.001$ and $\mathrm{P}<0.001$, respectively) (10). Furthermore, the objective response rate of the pembrolizumabchemotherapy combination was $64.1 \%$ in patients with tumors exhibiting PD-L1 expression on $\geq 50 \%$ of the tumor cells, significantly higher than that of pembrolizumab monotherapy (10). The mechanism behind this improved treatment response has been attributed in part to synergetic effects of chemotherapy's ability to cause cell death and immunotherapy's ability to improve the immune response. With the large amount of tumor cell death caused by the chemotherapy, many cancer antigens are released and taken in by the immune system for processing and immune response (boosting host immunity). The addition of immunotherapy agents can help prime and stimulate the immune response by removing immune checkpoints present in the tumor, thus improving T-cell activity. In the case of PD-1 blockade, the inhibition of this pathway results in less T-cell anergy and apoptosis, allowing for a more numerous and active T-cell response. In addition, it is believed that chemotherapy may create a more pro-inflammatory state by depleting immunosuppressive $\mathrm{T}$ cells and myeloid derived suppressor cells (MDSCs) (11,12). This mechanism is also proposed to be behind possible additive effects of PD-1 blockade and radiotherapy (13).

Tumor cells may utilize multiple immune checkpoints concurrently to evade the immune system and PD-L1 may be just one of them. Even if a single checkpoint is targeted and inhibited, the others still present may allow for immune escape and multi-target treatment would be required to overcome these resistance mechanisms. Creation of an immunosuppressive microenvironment can be achieved through the elaboration of molecules that decrease T-cell response or recruit immunosuppressive cells such as suppressor T-cells and MDSCs. These cells and factors may prevent T-cell activity and create a state of T-cell exhaustion, where T-cells are unable to generate an effective immune response. Thus, multiple combinations of PD-1 and another immune inhibitory molecule blockade have been studied. For instance, early studies of PD-1 and CTLA4 dual blockade have shown some initial promise in NSCLC $(14,15)$. Similarly, several therapies combining immunotherapy with antiVEGF treatment are being conducted with promising early results in multiple types of carcinoma, including NSCLC (16). At the step of tumor killing in the tumor microenvironment, multiple other immune checkpoints/ inhibitory molecules may exist and hamper the effects of PD-1 blockade. Indoleamine 2,3 di-oxygenase 1 (IDO1), one of such molecules, is an enzyme that catalyzes the metabolism of tryptophan to breakdown products, creating an immunosuppressive environment through depleting the local supply of tryptophan for lymphocytes and possibly through the creation of immunosuppressive molecules. The overexpression of IDO1 has been demonstrated in NSCLC and several drugs are currently in trials to determine their potential efficacy $(17,18)$. Lymphocyte activation gene 3 (LAG-3), $\mathrm{T}$ cell immunoglobulin and immunoreceptor tyrosinebased inhibitory motif (ITIM) domain (TGIT), and T-cell immunoglobulin and mucin domains (Tim)-3 and 4 have been identified as additional immune checkpoint molecules that may have potential as future targets for combination immunotherapy $(19,20)$. Similarly, B7-H3, a member of the B7 superfamily, has recently been added to the list of target candidates for combination immunotherapy for NSCLC (21).

\section{B7-H3 as a major immune checkpoint molecule in NSCLC}

The B7 superfamily of immunomodulatory molecules play a critical role in immunology by providing co-stimulatory signals to T-cells in the case of B7-1 and B7-2 binding to CD28. Although this is the canonical function of the B7 proteins, further research has shown that these proteins have multiple complex roles based on protein subtype, cell of expression, and binding ligand, and many of these may act to cause immunosuppression. This is particularly true for $\mathrm{B} 7-\mathrm{H} 4$ and $\mathrm{B} 7-\mathrm{H} 3$, which have both been implicated in tumor immune system evasion in numerous tumors $(22,23)$. Of those, $\mathrm{B} 7-\mathrm{H} 3$ is expressed by APCs and at low levels in other tissues. B7-H3 overexpression has been reported in a large number of tumors (22), and numerous studies have shown that it may inhibit $\mathrm{T}$-cell proliferation, cause reduced cytokine production, increase metastatic potential, and possibly inhibit tumor cell apoptosis $(22,24)$.

B7-H3 expression in NSCLC is common (ranging from between $32 \%$ to $70 \%$, depending on the study and cutoffs 
used) and is associated with more aggressive behavior, such as lymph node metastasis and advanced stage (25). One study using multiplex quantitative immunofluorescence found that $80 \%$ of NSCLC cases expressed $\mathrm{B} 7-\mathrm{H} 3$ and that a high $\mathrm{B} 7-\mathrm{H} 3$ expression was correlated with reduced survival but not with CD20, CD3, or CD8-positive tumorinfiltrating lymphocytes (TILs) (25). Numerous studies of $\mathrm{B} 7-\mathrm{H} 3$ in other tumor types have shown that $\mathrm{B} 7-\mathrm{H} 3$ is commonly overexpressed and is correlated with tumor aggressiveness and worse prognosis (24). Several B7-H3 antagonists are currently in development and early trials but conclusive clinical data is not yet available.

In the recent study by Yonesaka et al., the authors demonstrated that B7-H3 was expressed at some level (as determined by IHC) in $76 \%$ of their cohort of 82 NSCLC patients, and was higher in squamous cell carcinoma $v s$. non-squamous cell carcinoma ( $81 \%$ vs. $69 \%)$ and lower in patients with EGFR mutations vs. EGFR wild type (47\% vs. $81 \%$ ) (21). The expression of B7-H3 and PD-L1 appeared independent and the rate of $\mathrm{B} 7-\mathrm{H} 3$ positivity was higher in tumors that were resistant to anti-PD-1 therapy compared to those who responded to treatment (97\% in non-responders $v s .63 \%$ in responders). In addition, tumors lacking $\mathrm{B} 7-\mathrm{H} 3$ expression appeared to respond better to PD-1 blockade ( $88 \%$ versus $40 \%$ in tumors with any level of $\mathrm{B} 7-\mathrm{H} 3$ expression). The lack of $\mathrm{B} 7-\mathrm{H} 3$ expression was more predictive of response to PD-1 blockade than positive PD-L1 expression (OR/P value for response 17.5/0.0031 for B7-H3 vs. 0.794/0.754 for PD-L1). Expression of B7$\mathrm{H} 3$ and/or PD-L1 was strongly related to PFS in treated patients (HR/P value for PFS $0.082 / 0.003$ for B7-H3 vs. 3.6/0.006 for PD-L1), although $\mathrm{B} 7-\mathrm{H} 3$ provided better survival stratification of Kaplan-Meyer curves $(\mathrm{P}=0.024$ vs. 0.096). This study also found that PD-L1 was correlated to a higher number of CD8+ TILs whereas $\mathrm{B} 7-\mathrm{H} 3$ was correlated to a lower number of CD8+ TILs in the tumors with PD-L $1<50 \%$. From these findings, the authors theorized that $\mathrm{B} 7-\mathrm{H} 3$ expression may contribute to an immune tolerant phenotype through interference with CD8+ T-cell activity, and therefore be associated with resistance to PD-1 blockade therapy.

In order to further elucidate these findings, the authors performed mouse studies with a B7-H3-positive pancreatic cancer cell line (Pan02) and a B7-H3-positive lung cancer cell line (3LL) treated with an anti-B7-H3 antibody. With the Pan02 cell line, anti-B7-H3-treated mice had significantly smaller tumors compared to isotype controls, with a significantly increased number of both CD8+ and
CD4+ TILs and fewer FoxP3+/CD4+ (regulatory) TILs. In addition, treatment was associated with increased IFNgamma production from both CD8+ TILs and splenic lymphocytes, indicating increased T-cell activity. Treatment effect was nullified by CD8+ cell depletion but not CD4+ depletion. Taken together this implies that B7-H3 blockade may exhibit anti-tumor effects through reactivation of immune microenvironment, primarily mediated by CD $8+$ effector cells. When they repeated a subset of these experiments with the B7-H3-positive 3LL lung cancer cell line, they found that anti-B7-H3 antibodies reduced tumor growth with an increased number and activity of CD8+ TILs.

Finally, the authors explored combining PD-1 and B7$\mathrm{H} 3$ blockades in the previously tested Pan02 cell line (which is also PD-L1 positive). The tumors initially responded to both anti-PD-1 and anti-B7-H3 monotherapy but both developed resistance at around 31 days. However, combination therapy significantly inhibited tumor growth past day 39, completely eliminating the tumors in 2 of 6 mice and doubling survival time compared a control group. Combination blockade also resulted in a greater increase of CD8+ TILs compared to either single agent blockade. In addition, combination treatment did not appear to have any significant signs of toxicity in the animals treated. Of note, anti-B7-H3 monotherapy did not significantly alter PDL1 expression in the tumor cells, thus effects of the dual blockade are less likely attributed to enhanced PD-1/PD$\mathrm{L} 1$ axis by $\mathrm{B} 7-\mathrm{H} 3$ blockade.

This study elegantly demonstrated the potential of $\mathrm{PD}-1$ and $\mathrm{B} 7-\mathrm{H} 3$ dual blockade as combination immunotherapy for NSCLC but has several limitations that are worth mentioning. The human tissue study was limited in size $(n=82)$ and was not sub-classified by initial resection or post-treatment sample, nor by primary versus metastatic sample. In the majority of patients, the samples were from initial diagnosis and most patients treated with PD-1 blockade received it as $2^{\text {nd }}$ line or later, meaning that intervening treatment may have affected $\mathrm{B} 7-\mathrm{H} 3$ and PD-L1 expression as well as TIL populations. Of the 50 patients that received PD-1 blockade there was a response rate of $61 \%$, which is higher than many other studies, raising the possibility of patient selection bias. The animal cell line models were primary based on a pancreatic adenocarcinoma line Pan02, although the one experiment with the murine lung cancer cell line 3LL seemed to have a similar response to anti-B7-H3 treatment. Given these issues, further studies with larger populations of patients treated in a similar 
fashion and/or multiple lung cancer cell lines are warranted to confirm the findings of this study.

In summary, B7-H3 was commonly expressed and associated with less CD8+ TILs and a worse response to PD-1 blockade in this cohort of NSCLC patients. In animal models, $\mathrm{B} 7-\mathrm{H} 3$ blockade resulted in reduced tumor growth and increased CD8+ TILs. However, in both B7-H3 blockade and PD-1 blockade as monotherapy, the response was short-lived, while the combination of PD-1 and B7H3 blockade appeared to result in substantially improved response and durability. The high rate of expression and significant association of $\mathrm{B} 7-\mathrm{H} 3$ with $\mathrm{PD}-1$ blockade failure suggests that it may be a useful marker for patients that may not respond well to single-agent PD-1 blockade and represents a potential target for future treatments, in particular, combination therapy. Studies such as this are critical for characterizing these immune checkpoint molecules to take immunotherapy to the next level of efficacy.

\section{Acknowledgments}

Funding: None.

\section{Footnote}

Provenance and Peer Review: This article was commissioned and reviewed by the Section Editor Wei Xu (Jiangsu Provincial Key Laboratory of Geriatrics, Department of Geriatrics, the First Affiliated Hospital with Nanjing Medical University, Nanjing, China).

Conflicts of Interest: Both authors have completed the ICMJE uniform disclosure form (available at http://dx.doi. org/10.21037/tcr.2018.08.08). M Mino-Kenudson has served as a consultant for Merrimack Pharmaceuticals and $\mathrm{H} 3$ Biomedicine. MW Rosenbaum has no conflicts of interest to declare.

Ethical Statement: The authors are accountable for all aspects of the work in ensuring that questions related to the accuracy or integrity of any part of the work are appropriately investigated and resolved.

Open Access Statement: This is an Open Access article distributed in accordance with the Creative Commons Attribution-NonCommercial-NoDerivs 4.0 International License (CC BY-NC-ND 4.0), which permits the non- commercial replication and distribution of the article with the strict proviso that no changes or edits are made and the original work is properly cited (including links to both the formal publication through the relevant DOI and the license). See: https://creativecommons.org/licenses/by-nc-nd/4.0/.

\section{References}

1. Jemal A, Ward EM, Johnson CJ, et al. Annual Report to the Nation on the Status of Cancer, 1975-2014, Featuring Survival. J Natl Cancer Inst 2017;109. doi: 10.1093/jnci/djx030.

2. Chen DS, Mellman I. Oncology meets immunology: the cancer-immunity cycle. Immunity 2013;39:1-10.

3. Motz GT, Coukos G. Deciphering and reversing tumor immune suppression. Immunity 2013;39:61-73.

4. Tartour E, Pere H, Maillere B, et al. Angiogenesis and immunity: a bidirectional link potentially relevant for the monitoring of antiangiogenic therapy and the development of novel therapeutic combination with immunotherapy. Cancer Metastasis Rev 2011;30:83-95.

5. Reck M, Rodriguez-Abreu D, Robinson AG, et al. Pembrolizumab versus Chemotherapy for PD-L1Positive Non-Small-Cell Lung Cancer. N Engl J Med 2016;375:1823-33.

6. Borghaei H, Paz-Ares L, Horn L, et al. Nivolumab versus Docetaxel in Advanced Nonsquamous Non-Small-Cell Lung Cancer. N Engl J Med 2015;373:1627-39.

7. Herbst RS, Baas P, Kim DW, et al. Pembrolizumab versus docetaxel for previously treated, PD-L1-positive, advanced non-small-cell lung cancer (KEYNOTE-010): a randomised controlled trial. Lancet 2016;387:1540-50.

8. Fehrenbacher L, Spira A, Ballinger M, et al. Atezolizumab versus docetaxel for patients with previously treated non-small-cell lung cancer (POPLAR): a multicentre, open-label, phase 2 randomised controlled trial. Lancet 2016;387:1837-46.

9. Rittmeyer A, Barlesi F, Waterkamp D, et al. Atezolizumab versus docetaxel in patients with previously treated non-small-cell lung cancer (OAK): a phase 3, openlabel, multicentre randomised controlled trial. Lancet 2017;389:255-65.

10. Gandhi L, Rodriguez-Abreu D, Gadgeel S, et al. Pembrolizumab plus Chemotherapy in Metastatic NonSmall-Cell Lung Cancer. N Engl J Med 2018;378:2078-92.

11. Marmarelis ME, Aggarwal C. Combination Immunotherapy in Non-small Cell Lung Cancer. Curr Oncol Rep 2018;20:55.

12. Wargo JA, Reuben A, Cooper ZA, et al. Immune Effects 
of Chemotherapy, Radiation, and Targeted Therapy and Opportunities for Combination With Immunotherapy. Semin Oncol 2015;42:601-16.

13. Takamori S, Toyokawa G, Takada K, et al. Combination Therapy of Radiotherapy and Anti-PD-1/PD-L1 Treatment in Non-Small-cell Lung Cancer: A Minireview. Clin Lung Cancer 2018;19:12-6.

14. Hellmann MD, Ciuleanu TE, Pluzanski A, et al. Nivolumab plus Ipilimumab in Lung Cancer with a High Tumor Mutational Burden. N Engl J Med 2018;378:2093-104.

15. Hellmann MD, Rizvi NA, Goldman JW, et al. Nivolumab plus ipilimumab as first-line treatment for advanced nonsmall-cell lung cancer (CheckMate 012): results of an open-label, phase 1, multicohort study. Lancet Oncol 2017;18:31-41.

16. Herbst RS, Martin-Liberal J, Calvo E, et al. Interim safety and clinical activity in patients with advanced NSCLC from a multi-cohort phase 1 study of ramucirumab (R) plus pembrolizumab (P). Ann Oncol 2016;27:2800543.

17. Gangadhar TC, Schneider BJ, Bauer TM, et al. Efficacy and Safety of Epacadostat Plus Pembrolizumab Treatment of NSCLC : Preliminary Phase I/II Results of ECHO202/Keynote-037. J Clin Oncol 2017;35:9014.

18. Schalper KA, Carvajal-Hausdorf D, McLaughlin J, et al. Differential Expression and Significance of PD-L1, IDO-
1, and B7-H4 in Human Lung Cancer. Clin Cancer Res 2017;23:370-8.

19. Grosso JF, Goldberg MV, Getnet D, et al. Functionally distinct LAG-3 and PD-1 subsets on activated and chronically stimulated CD8 T cells. J Immunol 2009;182:6659-69.

20. Sakuishi K, Apetoh L, Sullivan JM, et al. Targeting Tim-3 and PD-1 pathways to reverse T cell exhaustion and restore anti-tumor immunity. J Exp Med 2010;207:2187-94.

21. Yonesaka K, Haratani K, Takamura S, et al. B7-H3 Negatively Modulates CTL-Mediated Cancer Immunity. Clin Cancer Res 2018;24:2653-64.

22. Li G, Quan Y, Che F, et al. B7-H3 in tumors: friend or foe for tumor immunity? Cancer Chemother Pharmacol 2018;81:245-53.

23. Zou W, Chen L. Inhibitory B7-family molecules in the tumour microenvironment. Nat Rev Immunol 2008;8:467-77.

24. Castellanos JR, Purvis IJ, Labak CM, et al. B7-H3 role in the immune landscape of cancer. Am J Clin Exp Immunol 2017;6:66-75.

25. Wu S, Zhao X, Wu S, et al. Overexpression of B7$\mathrm{H} 3$ correlates with aggressive clinicopathological characteristics in non-small cell lung cancer. Oncotarget 2016;7:81750-6.

Cite this article as: Rosenbaum MW, Mino-Kenudson M. Immunotherapy in non-small cell lung cancer: steps towards more effective combination therapies. Transl Cancer Res 2018;7(Suppl 7):S774-S778. doi: 10.21037/tcr.2018.08.08 